\title{
The Unity of Dependence
}

ABSTRACT: Most philosophers treat ontological dependence and metaphysical dependence as distinct relations. A number of key differences between the two relations are usually cited in support of this claim: ontological dependence's unique connection to existence, differing respective connections to metaphysical necessitation, and a divergence in their formal features. Alongside reshaping some of the examples used to maintain the distinction between the two, I argue that the additional resources offered by the increased attention the notion of grounding has received in recent years potentially offer us a way to unite the two relations, promising the attendant benefits parsimony offers, as a result.

KEYWORDS: ontology, grounding, metaphysics, dependence

\section{The Unity of Dependence}

Ontological dependence is usually characterized as being the relation at issue when something depends for its existence on the existence of another entity. For example, the claim ' $\{$ Socrates\} exists because Socrates exists' seems like a paradigmatic claim of ontological dependence. Fabrice Correia has given the following description in line with this characterization: '[O]ntological dependence is sometimes thought of as marking a certain form of ontological "non-self-sufficiency". A dependent object, so the thought goes, is an object whose ontological profile, e.g. its existence or its being the object that it is, is somehow derivative upon facts of certain sorts-be they facts about other particular objects or not' (2008: IOI3).

Along with ontological dependence, there is a wide variety of other types of non-causal dependence. The most notable among these, perhaps, is the notion of grounding. Grounding has received a great deal of attention recently (Rosen 20IO; Fine 20I2). It is usually taken to be the relation at issue in cases in which something exists or is a certain way in virtue of something else, among various other cognates (Clark and Liggins 2OI2). While most comfortable with the notion of grounding are happy to treat 'metaphysical dependence' and 'grounding' as synonymous, some-if not most-take ontological dependence to be distinct from grounding (Corkum 2016: \$4). Tuomas Tahko and E. Jonathan Lowe identify metaphysical dependence with grounding but distinguish grounding from ontological dependence (Tahko and Lowe 2016: \$5). Brian McLaughlin and

I am grateful to two anonymous referees, as well as the audience of the Ioth European Congress of Analytic Philosophy at the University of Utrecht, for comments on the final version of this article. I also thank David Liggins, Helen Beebee, Tuomas Tahko, Thomas Smith, Pilar Lopez-Cantero, and Abigail Connor for their input during the development of this work. 
Karen Bennett similarly distinguish ontological dependence from grounding (2018: $\$ 3.5)$. Correia treats 'ontological dependence' as standing for a 'non-well delineated, rich family of properties and relations' (2008: IOI3), rather than as a single relation. Despite this, it seems undeniable that there is at least some substantive connection between the two relations. As Henrik Rydéhn says, there is 'a great deal of overlap in the motivations typically appealed to when introducing them' (Rydéhn 202I: I232).

In this article, the position I take is that all instances of ontological dependence are a subset of grounding instances, and what marks an instance of grounding as an instance of ontological dependence is that it is an instance in which something's existence is that which is grounded. Contrary to the growing consensus among grounding theorists, identifying grounding with ontological dependence is indeed possible; the resulting position is coherent; and the position avoids some of the most obvious problems modal-existential accounts of ontological dependence face. The resulting simplification is desirable from the standpoint of parsimony in so far as it requires commitment to only one notion-grounding-rather than two (grounding and ontological dependence). Within the scope of this article, I argue only that the position is one worth taking seriously. More work remains to be done to establish the position as favorable in comparison with accounts of ontological dependence other than modal-existential accounts, for example, essentialist accounts of ontological dependence, like that of Tahko and Lowe (20I6).

Along the way, I argue toward two notable conclusions: (I) generic ontological dependence is best understood as reducible to rigid ontological dependence; and (2) factive and non-factive conceptions of dependence relations (be they grounding relations or otherwise) are perhaps best understood by bifurcating the notion of dependence, in a seemingly noncommitting manner.

In contrast to ontological dependence, the way grounding is usually treated, it need not concern an entity's existence, nor need it concern the essential features of that entity (on the assumption that something's essential features contribute to the existence of that thing). The contingent properties of an entity, for example, may metaphysically depend on some other properties had by that entity. The painting is beautiful because of the color composition, for example; ignoring those aestheticians who consider a work's aesthetic properties to be essential to it (Lamarque 2010: ch. 5), this claim of metaphysical dependence does not concern the existence of the painting, or the existence of the property beautiful, but the contingent token instantiation of a property/properties on which another token instantiation-its beauty-appears to depend.

Another way in which the two relations-ontological dependence and grounding - appear to differ is in their connection to metaphysical necessity. The orthodox view of grounding is that it is a metaphysically necessary relation; that is, if $x$ grounds $y$, then, necessarily, if $x$ obtains, $y$ obtains. Necessitarians-those who support the thesis that grounding carries metaphysical necessity-include Paul Audi (20I2), Ross Cameron (20I0), Kit Fine (20I2: 37), Gideon Rosen (2010), and D. Gene Witmer, William Butchard, and Kelly Trogdon (2005). Opponents of the view include Stephan Leuenberger (20I4) and Alexander Skiles (20I4) (see Trogdon 20I3: 476-78). On the other hand, it does not appear that ontological 
dependence carries such weight. Consider the following two examples adapted from Tahko and Lowe (20I6: $\mathbb{5} 5$ ): assume for the moment that water ontologically depends on hydrogen and oxygen. Despite this being the case, hydrogen and water could both exist and yet fail to combine in the appropriate structure to form water. It does not appear, then, that the existence of hydrogen and oxygen necessitate the existence of water, despite the fact that, by assumption, water ontologically depends on hydrogen and water. Similarly, it is plausible that children ontologically depend on their parents (Tahko and Lowe 2016: \$2), and yet it would be hard to maintain that the existence of a parent metaphysically necessitates the existence of their children. That someone has children or not is surely a contingent matter, even though a person's children are their ontological dependents.

Though the divergence on the connection to metaphysical necessity is probably the most significant difference between the two relations, there are other dissimilarities worth mentioning-though slightly easier to dispatch. First, ontological dependence is commonly taken to relate objects of multiple ontological categories, whereas grounding is standardly taken to relate facts alone (Rosen 20IO; Audi 20I2: I03). And even that is only if one agrees grounding talk is best regimented with the use of a predicate with which 'grounds' correspondsthat is to say that one is a predicationalist. For those that think grounding talk is best regimented by way of a non-truth-functional sentential operator, as the so-called operationalists have argued (Fine 20I2: 46; Correia 20IO: 3-4; Dasgupta 20I4: 3-4; and Schnieder 20II), the notion of a 'relatum of grounding' will be entirely foreign. I largely ignore this difference for now, however. Though the orthodox position among predicationalists is indeed that grounding relates facts only, there is opposition to the view (most notably, Jonathan Schaffer 2009). Importantly, such opposition is separately motivated from the matters under discussion in this section. I will assume for present purposes, then, that grounding can indeed relate more than facts, just as I will assume ontological dependence can.

Another difference between grounding and ontological dependence that has been pointed out is that the two have different formal features: Tahko and Lowe note that grounding is usually treated as an irreflexive relation (20I6: $\mathbb{S} 5$ ). A relation $R$ is irreflexive iff, for any $x$, there are no instances in which any $x$ bears $R$ to itself. Ontological dependence, they argue, need not be. Given differing formal features, the relations cannot be identical. In response to this argument I would note that the issue of the formal features of either relation is not a settled one. Responses have been offered to arguments that grounding is irreflexive (Jenkins 20II), and the position has been taken that ontological dependence is (Correia 2008: IO24). Tahko and Lowe themselves acknowledge in an earlier edition of the article that the existence of entities that ontologically depend on themselves-in violation of irreflexivity-'is of course controversial' (2015: \$5). Without its being clear that ontological dependence is indeed not irreflexive, and equally that grounding is, I take it that there is room-for present purposes, at least-to assume that the formal features of the two align.

Relatedly, the orthodox position on grounding is that it is an asymmetric relation (Rosen 2010: II 5-I6, Schaffer 2009: 376), while ontological dependence, it has been argued, is not (Barnes 20I8). A relation $R$ is asymmetric iff, for any $x$, there 
are no instances whereby $x$ bears $R$ to $y$ and $y$ bears $R$ to $x$. Elizabeth Barnes (20I 8 ) has argued that ontological dependence is not asymmetric. I concede that, to a large extent, I am operating here under the major conditional assumption that ontological dependence is not asymmetric. Below I briefly address part of Barnes's (20I8) argument that ontological dependence is not asymmetric - if only to demonstrate the conditional assumption I am operating under here is not preposterous. Barnes's full argument is compelling and complex (and outside the scope of this article), but I offer the reply to one particular strand of her argument in an attempt to assuage this concern.

Part of Barnes's argument for the symmetricality of ontological dependence concerns Armstrong's overarching metaphysical project (Armstrong I997). Her position is that the most plausible form of Armstrong's account of state of affairs -on which state of affairs are understood as a complex of property and particular-is one on which it is held that both (I) state of affairs depend on their constituents (particulars and properties) and (2) constituents depend on their state of affairs. As such, the Armstrongian requires a notion of dependence that is symmetric (with state of affairs being both dependent on, and ontological dependee of, their constituents, and vice versa). Her objection to holding only the first of these theses is that this ultimately places fundamentality with particulars and properties, which runs contrary to the Tractarian worldview in which the position is embedded (Barnes 20I8: 57), on which the world is one ultimately constituted by state of affairs. Thesis (I) states that, in effect, particulars and properties are the ultimate constituents of the world, rather than state of affairs. Her contention against holding only the second of these theses is that doing so means the position becomes 'explanatorily impoverished' (Barnes 201 8: 57). The argument is that we lack the resources with which to highlight commonalities and dissimilarities between states of affairs in only holding thesis (2). Why does John is a human resemble Mary is a human more closely than does Rex is a dog? Well, it does so because of shared constituents between the first and second state of affairs-namely, the property is a human. The property is a buman is a constituent, however, and thus relatively less fundamental to the state of affairs itself according to the second thesis. Barnes's contention is that if the ultimate explanatory bedrock is just the states of affairs, and not their constituents, then it is hard to see how we could explain this commonality' (2018: 57). I am not persuaded by this argument, as I am not sure as to why we should hold the position that the only method with which to recognize commonalities between states of affairs is by recourse to fundamentalia. It seems plausible that in other circumstances, we properly recognize commonalities between fundamental entities by recourse to non-fundamental commonalities. Imagine two identical physical states; barring cases of multiple realizability, we can infer from the identicality of macro-level features (that is, non-fundamental characteristics) that the microphysical states that underlie those features (that is, the fundamental) perfectly resemble each other as well-lest they give rise to differing macro-level features. Barnes seems to assume such identificatory practices are impossible; I see no obvious reason to think that they are. Barnes's overall contention is that we must hold both theses to be true to avoid the problem facing either, and as such, 
we require a notion of ontological dependence that is symmetrical (with particulars and properties depending on state of affairs, and vice versa); my reply is that it is not obvious the problem she has identified with the second thesis is a knockdown one, and as such, it is open to the Armstrongian to hold only the second thesis. As such, Barnes's argument does not demonstrate the necessity of symmetrical dependence.

Again, Barnes's case for the symmetricality of ontological dependence goes beyond this singular argument; however, this should suffice to convince that operating under the conditional assumption that ontological dependence is asymmetric is not a ridiculous position to take.

More troublingly for those who would identify grounding and ontological dependence, Tahko and Lowe also note that grounding appears to have a different connection to explanation than ontological dependence. They give the following example to demonstrate this difference: '[E]ven though the existence of water depends on the existence of hydrogen and oxygen, it does not seem to be the case that the existence of hydrogen and oxygen explain the existence of water. Rather, what explains the existence of water is the ability of hydrogen and oxygen atoms to form molecules (even though this is rather simplified)' (Tahko and Lowe 2016: \$5). Though water ontologically depends on hydrogen and water, so the thought goes, the existence of hydrogen and water does not explain the existence of water. (I leave this objection to one side for now but respond in full below in section 7.) So, prima facie, grounding and ontological dependence are distinct relations. A reconciliation is possible.

\section{Rigid and Generic Ontological Dependence}

Given that I propose we analyze ontological dependence in terms of grounding, it is important to have a solid grasp on what we are analyzing. It is commonly recognized that there are two distinct forms of ontological dependence: rigid ontological dependence and generic ontological dependence (Tahko and Lowe 20I6: \$I; Rydéhn 202I). Rigid ontological dependence is taken to be a one-to-one relation, holding between two specific entities. For example, assume that \{Socrates\} ontologically depends on Socrates. No other entity will suffice as the ontological base of $\{$ Socrates\}; Socrates is the only viable candidate. Various other plausible instances of ontological dependence, however, are more lenient, so to speak. That is, it appears multiple entities could play the role of the ontological dependee. For example, [someone is a philosopher] ${ }^{\mathrm{I}}$ ontologically depends not on any particular person, but on at least one person satisfying the predicate is a philosopher. The relation of ontological dependence, in this case, does not hold between a specific $x$ and a specific $y$, as in the case of \{Socrates\} and Socrates, but rather between a specific $x$ (in this case, [someone is a philosopher], and some $F$ (namely, whichever person happens to satisfy the predicate is a philosopher). When specific entities are

\footnotetext{
${ }^{\mathrm{I}}$ I use the standard notational convention of square brackets to stand for the fact that, and angled brackets to stand for the proposition that. For example, the fact that the ball is red would be symbolized as [the ball is red]. The proposition that the ball is red would be symbolized as <the ball is red $>$.
} 
related by ontological dependence, the relation at issue is usually taken to be one of rigid ontological dependence. When a specific entity ontologically depends for its existence on the existence of something-anything-that satisfies a certain predicate, the relation at issue is usually taken to be one of generic ontological dependence.

Given that the hallmark of ontological dependence appears to be something's requiring for its existence that something else exist, modal-existential analyses show initial promise as an analysis of the notion of ontological dependence. The following definitions (adapted from Tahko and Lowe 20I6: \$2) seem to capture what we want to say of ontological dependence. The first is a definition of rigid ontological dependence:

Rigid ontological dependence. $x$ ontologically depends $s_{\mathrm{r}}$ upon $y==_{\mathrm{df}}$.

Necessarily, $x$ exists only if $y$ exists.

This seems to align with what we want from our notion of dependence; that something cannot exist without that on which it ontologically depends existing. \{Pegasus\} ontologically depends on Pegasus, and so can only exist in worlds in which Pegasus exists, and the above definition captures this.

Similarly, the following definition seems to capture the notion of generic ontological dependence:

Generic ontological dependence. $x$ ontologically depends $\mathrm{g}_{\mathrm{g}}$ upon some $F$ $=_{\mathrm{df}}$. Necessarily, $x$ exists only if some F exists.

The above definition seems to capture what we want for generic ontological dependence. To use my earlier example, [someone is a philosopher] exists only as long as someone satisfies the predicate is a philosopher.

At this point, it is necessary to switch briefly back to the notion of grounding to consider the notion of factivity. Fine has considered the matter of whether grounding is factive, giving the following statement as to when a conception of grounding is factive or non-factive:

On the factive conception, we can only correctly talk of something factive-such as a true statement or a fact-being grounded; and what grounds must likewise be factive. But on the non-factive conception, we can also correctly talk of something non-factive-such as a false statement or a merely possible fact-being grounded; and what grounds may likewise be non-factive. Thus on the factive conception, $\mathrm{A} \wedge \mathrm{B}$ can only be grounded in $\mathrm{A}$ and $\mathrm{B}$ if $\mathrm{A} \wedge \mathrm{B}$ (and hence $\mathrm{A}$ and $\mathrm{B}$ ) are indeed the case while, on the non-factive conception, $A \wedge B$ can be grounded in $\mathrm{A}$ and $\mathrm{B}$ even if $\mathrm{A}$ or $\mathrm{B}$ (and hence $\mathrm{A} \wedge \mathrm{B}$ ) is not the case. (Fine 20I 2: 48-49)

If one takes a factive view of grounding, one is committed to the view that grounding only holds between actual existents. If one takes a non-factive view, one allows for 
the holding of grounding relations in worlds other than the actual one; the relata (if indeed one takes grounding to be a relation) need not actually exist for a grounding relation to hold between them. It is worthwhile to note here that throughout this article I talk of grounding-and ontological dependence, for that matter-as being relational. I do so only for convenience. Nothing hinges on this assumption, and the same arguments mutatis mutandis could be made for those who treat grounding as an operator-or similarly do not think of ontological dependence as a relation.

To switch back to modal existential analyses of ontological dependence: as defined above, both rigid and generic ontological dependence are non-factive. To take the case of rigid ontological dependence, as long as modal space is configured such that in all worlds in which $x$ exists, $y$ exists, then $x$ depends $_{\mathrm{r}}$ on $y$, even if $x$ and $y$ do not exist in the actual world. \{Pegasus\} exists only in worlds in which Pegasus exists, but that is a fact about all possible worlds, not solely about this world. As such, defined modally, ontological dependence appears to be non-factive.

\section{Problems for Modal Existential Definitions}

To cut to the chase, then, why should we reject non-factive, modal existential definitions of ontological dependence?

Firstly, there are now familiar problems that parallel the problems with identifying supervenience-another modal term-with ontological dependence.

Necessary existents pose a problem for modal-existential analyses of ontological dependence. In all worlds, we might suppose, the number two exists. Everything coexists with the number two. This means, however, that according to modal-existential definitions of ontological dependence such as the one given above, everything depends $s_{\mathrm{r}}$ on the number two. This is surely incorrect. However one might define ontological dependence, we should surely not prefer a definition that would have us commit to the view that everything depends, ontologically, on the existence of the number two. More conservatively, we should be persuaded to this surprising conclusion only by additional argumentation toward the truth of this implication. Pretheoretically, the claim that everything depends on the existence of the number two is an extraordinary claim, and it is precisely because it is an extraordinary claim that it requires additional argumentation. This additional argumentation, to my knowledge, has never been offered by any advocate of the modal-existential analysis. As such, an account of ontological dependence that does not require this extraordinary commitment will be more attractive than one that does, all else being equal.

Options for avoiding the problem of necessary existents include restricting the application of ontological dependence to contingent existents only. Simons (I987: 295) suggests just such a move. The problem with restricting the notion in this way, however, is that it looks ad hoc unless a principled reason is given why necessary existents cannot depend or be depended upon, ontologicallysomething that Simons, for one, does not do.

Even if such a move were granted, however, there is still the problem of contingently existent coexistent entities in which dependence runs only one way. 
\{Socrates\} exists in all worlds that Socrates does and does not exist in any worlds in which Socrates does not; as a result, \{Socrates\} depends $s_{r}$ on Socrates. The problem, however, is that in all worlds in which Socrates exists, so, too, does \{Socrates\}. As a result, according to the definition, Socrates depends $\mathrm{r}_{\mathrm{r}}$ on \{Socrates\} as well.

This material has been well covered in the context of arguments against supervenience, so I will not labor the point too much. Modal existential analyses of dependence generally will not suffice for the reasons above. A less explored problem for modal existential analyses is that it is arguable that they get a lot of dependence talk wrong. Consider the following example: according to the modal-existential definition, \{Pegasus\} depends $s_{\mathrm{r}}$ on Pegasus. Furthermore, that dependence holds in our world. As long as Pegasus and \{Pegasus\} coexist in all worlds, $<$ Pegasus $\}$ depends $\mathrm{r}_{\mathrm{r}}$ on Pegasus $>$ is true at the actual world, even if \{Pegasus\} and Pegasus do not actually exist in the actual world, according to the above definition. Modal existential analyses of ontological dependence say something about the configuration of modal space-in this case, that Pegasus and \{Pegasus\} coexist in the worlds that constitute modal space. The statement is true when uttered from anywhere within that modal space-for example, when uttered in the actual world. This means that the modal existential definition of ontological dependence will commit us to the truth of a host of dependence claims that we would probably prefer to say are, strictly speaking, false. Pretheoretically, it seems correct to say that \{Pegasus\} does not depend on Pegasus in the actual world, given that neither actually exist, though according to the definition of rigid ontological dependence as stated above, this relation of dependence does indeed hold in the actual world. This can be construed as an issue of fineness of grain. Strictly speaking, dependence claims regarding actual non-existents should turn out as false when that claim is made about the actual world. Even if $x$ depends $_{\mathrm{r}}$ on $y$ in worlds other than our own, it should surely turn out that, when speaking carefully, the claim that $x$ does not depend on $y$ in our world is true, if neither $x$ nor $y$ exist in our world. All else being equal, we should prefer an account that allows us to make the distinction between actual instances of ontological dependence and merely possible instances of dependence over an account that blurs this distinction.

This argument differs from a similar one that Rydéhn considers and responds to (202I: I 245). He argues that, though it is true that (non-intensional) relations cannot hold between nonexistents, there is no reason to think of ontological dependence as a relation. Thus, the argument that the modal existential analysis of ontological dependence should be avoided because it entails that relations hold between non-existents-such as \{Pegasus\} and Pegasus-fails. Although I think of ontological dependence as relational, I am happy to grant the assumption that ontological dependence is non-relational for current purposes. My argument differs from that which Rydéhn responds to-and thus still applies-in that it rests solely on linguistic evidence: regardless of whether ontological dependence is a relation or not, we should be uncomfortable with a definition of ontological dependence that commits us to the truth of statements such as $<$ Pegasus\} ontologically depends on Pegasus $>$ in the actual world. An account of ontological dependence should afford us resources that are sufficiently fine-grained as to distinguish between claims of dependence that hold in the actual world, and 
claims regarding merely possible entities. The modal-existential analysis appears to fail, in this regard.

A different response that might work is to modify the definitions in the following manner:

Actual rigid ontological dependence. $x$ actually depends $\mathrm{r}_{\mathrm{r}}$ on $y=\mathrm{df}$. Necessarily, $x$ exists only if $y$ exists, and $x$ and $y$ actually exist.

Possible rigid ontological dependence. $x$ possibly depends $\mathrm{s}_{\mathrm{r}}$ on $y=_{\mathrm{df}}$. Necessarily, $x$ exists only if $y$ exists, and $x$ and $y$ exist in at least one world.

By bifurcating the notion of rigid ontological dependence in the manner abovemutatis mutandis for generic ontological dependence-we can align with our intuitions regarding dependence talk. \{Pegasus\} only possibly depends $\mathrm{s}_{\mathrm{r}}$ on Pegasus, according to the above definition, in the actual world, given that neither \{Pegasus\} nor Pegasus actually exist, and given that they do coexist in all possible worlds in which Pegasus exists. Thus, statements such as $<$ \{Pegasus $\}$ ontologically depends on Pegasus > will now turn out as false at our world, if the statement is understood to mean that \{Pegasus\} actually depends $\mathrm{r}_{\mathrm{r}}$ on Pegasus. Similarly, as long as in all possible worlds where \{Pegasus\} exists, Pegasus exists, and the actual world is not among those possible worlds, then \{Pegasus\} only possibly depends $s_{r}$ on Pegasus. Propositions such as <Though \{Pegasus\} does not actually depend on Pegasus, had \{Pegasus\} existed, it would depend on Pegasus $>$ turn out as true in worlds in which neither \{Pegasus\} nor Pegasus exist, as long as they coexist in all other possible worlds.

Whether or not this move assuages the concern I have raised, the foregoing issues with modal existential analyses of dependence, as Tahko and Lowe say, 'nevertheless leave room for an alternative account' (Tahko and Lowe 20I6: $\$ 4$.I). Whereas Tahko and Lowe go on to consider essentialist accounts of ontological dependence, I argue for an account of ontological dependence in terms of grounding.

\section{Ontological Dependence as Grounding}

As stated above, my proposal is that we take instances of ontological dependence to be instances of grounding. More specifically, instances of ontological dependence are a subset of grounding instances, marked by their concerning existence solely. That is, any instance of grounding which concerns something's existing in virtue of another, distinct thing's existence is a case of ontological dependence. Instances of ontological dependence are a subset of instances of grounding as there are many other instances of grounding that do not concern existence. For example, though a painting's aesthetic qualities might be grounded by its nonaesthetic properties, this instance of grounding need not concern the existence of those aesthetic qualities, per se (as long as one is not a trope theorist, in which case the claim might be more credible). This suggests the following definition of ontological dependence:

Grounding rigid ontological dependence. $x$ ontologically depends on $y=\mathrm{df} . x$ 's existence is grounded by the existence of $y$. 
This definition appears to capture correctly what we want from the notion of ontological dependence. It is fairly straightforward to see why. Briefly, to take the example of \{Pegasus\} and Pegasus once more: \{Pegasus\}'s existence is grounded by the existence of Pegasus, and so, $\{$ Pegasus $\}$ is said to ontologically depend on Pegasus.

By fleshing out the notion of ontological dependence in terms of grounding, we avoid the problems presented by necessary existents and coexistent contingent existents. Despite the fact that everything coexists with the number two, not everything is grounded by the number two, and thus not everything ontologically depends on the number two, as the unrestricted modal existential analysis forced us to accept. Similarly, necessary coexistents no longer cause a problem: though Socrates and \{Socrates\} coexist in all worlds, it is \{Socrates\}'s existence that is grounded by the existence of Socrates, and not the other way around. Therefore \{Socrates\} ontologically depends on Socrates, and not vice versa.

One issue with the proposal is that, subject to one's position on the factivity of grounding, once again the notion might not align with how we talk about ontological dependence. If we take a factive view of grounding, on which we can correctly speak only of grounding relations that actually hold, we might lack the tools by which to make claims about merely possible instances of ontological dependence. If \{Pegasus\} and Pegasus do not exist, on the factive view the two cannot be related by grounding. As such, $<$ Had \{Pegasus\} existed, it would ontologically depend on Pegasus> turns out as false. This might suggest support for the non-factive view, as the problem does not arise in terms of non-factive grounding; given that there are possible worlds in which \{Pegasus\}'s existence is grounded by Pegasus's existence. This suggests a similar bifurcation of the notion in the same manner as modal existential definitions:

Actual grounding rigid ontological dependence. $x$ actually ontologically depends on $y=_{\mathrm{df}}$. $x$ 's existence is grounded by $y$ 's existence in the actual world.

Possible grounding rigid ontological dependence. $x$ possibly ontologically depends on $y==_{\mathrm{df}}$. $x$ 's existence is grounded by $y$ 's existence in at least one world, and not the actual one.

Here is not the place to delve into factivity in any great depth; I merely mark it as worthy of attention. It is also worth noting that the issue of the factivity of grounding has not received a massive amount of attention. (For more, see Rydéhn 202 I; Fine 20I2: 52; Leuenberger 20I4: 232). For the time being, I will assume the non-factive view of grounding, on which grounding relations can be correctly said to hold in worlds other than our own, though admittedly primarily for the pressure presented by this case.

\section{Problems with Identifying Grounding with Ontological Dependence}

In the previous section, I did not supply two distinct definitions for rigid and generic ontological dependence. I did not do so because, as Rydéhn forcefully argues (202 I: 
I 253-I 254), grounding looks dissimilar from generic ontological dependence in one important respect, as I discuss below. It is on the basis of this dissimilarity that Rydéhn argues that generic ontological dependence is not sufficient for non-factive grounding. Given that I am arguing that ontological dependence is grounding, this obviously poses a significant problem for the position I have taken.

This brings me neatly to the first of two problems that I respond to in the following sections. The problem, then, is the apparent inability of grounding to properly capture generic ontological dependence. The orthodox position assumed in the grounding literature appears to be that grounding always relates specific entities; the formulations of grounding claims one usually sees are usually variations of the form ' $y$ is grounded by $x$ '. What is not seen, however, are variations of the form ' $y$ is grounded by some F', which would roughly correspond to the notion of generic ontological dependence. That being so, it does not seem that the notion of grounding is well suited to analyzing the notion of generic dependence. Given that grounding always relates specific entities, how then can it capture a notion on which a specific entity is said to depend on the satisfaction of a predicate? Of course, just because such formulations are not popular does not mean they are not legitimate. Perhaps future grounding theorists will indeed present arguments that the present construction of grounding claims should be expanded in a manner conducive to analyzing the notion of generic ontological dependence. However, erring, as Rydéhn does, on the side of caution, I agree to restricting grounding claims to those that hold between specific entities. Thus, we are left with a notion that appears to resemble rigid ontological dependence only. Why then, should we be convinced of an analysis that only correctly captures one half of the traditionally bifurcated notion of ontological dependence? This is not as problematic as it might first appear. We can reduce the notion of generic ontological dependence to rigid ontological dependence.

\section{Generic Ontological Dependence and Grounding}

Generic ontological dependence proves to be a particularly thorny notion to capture with the notion of grounding. We commonly see claims of the form $a$ ontologically depends on some F. For example, [something exists] ontologically depends on anything satisfying the predicate exists. Canonically, grounding instances are of the form $x$ grounds $y$, however. Rydéhn forcefully makes this point in arguing that generic ontological dependence is not sufficient for grounding (2O2I: I 235, I 242).

One might worry here that facts are not the sorts of things that ontologically depend. I would plead for leniency in this case and that the assumption that they do be granted for the time being, merely for the reason that the examples including facts are quite illustrative, and nothing hinges on the assumption that facts can enter into relations of ontological dependence (an assumption I would reject, at any rate). Similarly, I would also ask for leniency regarding the predicate exists; while some will not consider exists as a genuine predicate, I ask that this belief be parked for now to ensure my discussion remains idiomatic.

The problem, then, is claiming that instances of the form $x$ ontologically depends on some $F$ means that it cannot be an instance of grounding, given that grounding relates specific entities. 
We can avoid this problem by making the claim that there are no genuine instances of generic dependence. That is, we deny that ontological dependence ever really holds between an entity and a generic requirement for the satisfaction of a predicate. A promising means by which to do so is to claim instead that claims of generic ontological dependence are actually paraphrases of rigid ontological dependence. That is, despite appearances, when we make the claim that $x$ ontologically depends on some $F$, what we are actually doing is giving a paraphrase of all the instances of which $x$ is grounded by something by noting what is common to all the things that ground $x$ across worlds, namely that its grounds are all Fs.

For example, [someone is a philosopher] is grounded in various worlds, including the actual world, by a vast variety of people, all of whom happen to be philosophers. We can reduce our reporting of this case by citing that which is common to all these various people, namely, their instantiating the property is a philosopher. Nonetheless, there is no need to posit a direct grounding link between the property is a philosopher and [someone is a philosopher]. The grounding relation holds between each of the various persons (who individually instantiate the property is a philosopher) and the fact. And this is entirely satisfactory. That is, there is no need to leave it 'open ended', such that we allow for novel instantiations of the property to ground the fact. Modal space is such that everyone or everything that satisfies the predicate is a philosopher exists in at least one world in which they ground [someone is a philosopher].

The suggestion then is that we understand ' $x$ is grounded by some $F$ ' as a paraphrase of all the instances of rigid ontological dependence that hold between $x$ and its grounds across modal space. We then shorten this catalogue by simply noting what is common to all the things that ground $x$ across these worlds. Nonetheless, this is only a useful tool. Strictly speaking, there is only rigid ontological dependence.

One might reply here that it appears this endeavor is doomed to failure for the following reason: Take [someone is a philosopher]. Imagine in $\mathrm{W}_{\mathrm{I}}$ that this fact ontologically depends on [Sally is a philosopher]. Now imagine in $\mathrm{W}_{2}$ that this fact ontologically depends on [Bob is a philosopher]. According to the modal-existential analysis, [someone is a philosopher] cannot depend $\mathrm{d}_{\mathrm{r}}$ either on [Sally is a philosopher] or [Bob is a philosopher]. Why? Because it is not the case that [someone is a philosopher] coexists with either fact in all worlds; in $\mathrm{W}_{\mathrm{I}}$ [Bob is a philosopher] does not obtain, and in $\mathrm{W}_{2}$ [Sally is a philosopher] does not obtain-highlighting the fact there is no necessary coexistence between the facts. How, then, can we maintain that [someone is a philosopher] rigidly depends on definite, specific facts, such as [Sally is a philosopher], as I have claimed? The answer is that, by abandoning the modal-existential analysis of ontological dependence, this situation does not pose a problem. As long as [Sally is a philosopher] grounds [someone is a philosopher] in $\mathrm{W}_{\mathrm{I}}$, then [someone is a philosopher] can be said to ontologically depend on [Sally is a philosopher], according to the definition given above. Untethered from the modal-existential analysis, we no longer require necessary coexistence for ontological dependence, and as such, the above situation poses no problem. 
Given a factive view of grounding, speaking carefully, $x$ is not grounded by anything other than the philosophers in the actual world. The factive view of grounding precludes us from holding that grounding claims about grounding in other worlds than our own are true. We can only include instances of our own world in the paraphrase, then. This should give us reason for concern, so the thought goes: we surely want instances other than our own (merely possible satisfactions of some $F$ ) to ground $x$. I am convinced by this objection. Helpfully, on the non-factive conception of grounding, the problem does not appear. We can speak correctly of $x$ 's being grounded by some $F$ in worlds other than our own on the non-factive view, as it allows us to speak correctly of grounding relations that only hold in possible worlds. As such, we can include the litany of merely possible grounding relations in our paraphrase, and the problem evaporates.

Understanding claims of generic ontological dependence as paraphrases of claims of rigid ontological dependence then allows us to bring it into the fold of grounding. Strictly speaking, there are no veridical instances of generic ontological dependence, and thus the dissimilarity between generic ontological dependence and grounding on the matter of specificity is no problem.

\section{Metaphysical Necessity, Ontological Dependence, and Grounding}

The second problem, as mentioned in the introduction, concerns the connection between ontological dependence and metaphysical necessity, or the lack thereof. Whereas grounding arguably entails metaphysical necessity, it does not appear that ontological dependence does. This is easily demonstrable by way of numerous plausible examples of ontological dependence in which the existence of the dependee does not appear to necessitate metaphysically the existence of the dependent. Children ontologically depend on their parents, though the existence of the parent does not necessitate the existence of a child. Hydrogen and oxygen are the ontological dependees of water, though their existence does not necessitate the existence of water-it is easy to imagine a world in which the two elements simply never happen to meet. If the two differ in their connection to metaphysical necessity, so goes the argument, then the two must be different relations.

The way around this problem is recognizing instances of ontological dependence as examples of partial grounding. The best way to illustrate the distinction between full and partial grounding appears to be by way of example (as does Fine 20I 2: 50). Socrates fully grounds \{Socrates\}, but only partially grounds \{Socrates, Plato\} (also see Bliss and Trogdon 2016: $\$ 5$ ). As mentioned earlier, the orthodox view among grounding theorists, and the one I will assume to be true here, is that grounding entails metaphysical necessitation; if $x$ grounds $y, x$ metaphysically necessitates $y$. This needs to be sharpened slightly to the claim that full grounding entails metaphysical necessitation. The consensus view on partial grounding is that partial grounding does not entail metaphysical necessitation (Leuenberger 2020: 2655; Skiles 20I4: 720; Trogdon 20I3: 467). It is easy to see the intuitive plausibility of this position; while Socrates is the full ground of $\{$ Socrates\}, and thus Socrates metaphysically necessitates \{Socrates\}, Socrates only partially 
grounds \{Socrates, Plato\}, and hence does not metaphysically necessitate it-only in concert with Plato does it do so, but Plato and Socrates together constitute the full grounds of $\{$ Socrates, Plato\}.

Given the primitive nature of grounding, cashing out the distinction between partial and full grounding is difficult. The distinction between full and partial grounds is often made in quite oblique terms. Clark says, for example, '[w]here a fact partially grounds another, the former helps to bring it about that the latter obtains, but may not suffice to do so. The notion of sufficing to bring about is the intuitive core to the notion of full grounding' (2015: I89). Similarly, Skiles distinguishes between the two by saying that partial grounds contribute to the full grounds (2014: 720). Leuenberger characterizes the orthodox account of the distinction as holding partial grounds to be part of the full grounds, going on to clarify that, "[u]sually, "part" is not understood in the strict mereological sense here' (Leuenberger 2020: 2656). The lack of metaphysical necessitation on the part of partial grounds seems to be one significant mark by which to distinguish the two, and I will assume this to be true for our current purposes. Two slight complications here: firstly, Trogdon has noted the example of the grounding of necessary truths provide a counter to this claim (Trogdon 20I3: 467). Briefly: for a conjunction of two or more necessary truths, any individual conjunct metaphysically necessitates the conjunction despite only partially grounding it, given that the conjunction is metaphysically necessary anyway. We can ignore this tangential complication for what follows, assuming for the moment that the nature of this problem is based in the peculiarity of metaphysically necessary truths. Secondly, something worth mentioning here is that the consensus view is that all instances of full grounding are instances of partial grounding (but not vice versa) (Leuenberger 2020: 2654; Fine 20I2; Schaffer 20I2: I 27). A helpful notion here, from Leuenberger, is that of merely partial grounds; a partial ground that is not also a full ground. He uses an example to illustrate the difference: ' $[\mathrm{M}] \mathrm{y}$ having a mass is a partial ground for my attracting the earth. But it is a merely partial ground, since a full ground would also include further facts, for example the earth's having mass and the holding of the law of gravitational attraction' (Leuenberger 2020: 2655).

Apart from cases in which there appears to be grounding, but no metaphysical necessity, it appears we have to take it as a brute fact that some instances of grounding are partial, and some of those are full, and that we can recognize these cases when we see them. The point to be taken away here, however, is that partial grounds need not metaphysically necessitate that which they partially ground.

Examples of ontological dependence, then, may sometimes be examples of merely partial grounding. That being the case, we should naturally expect that ontological dependence does not always entail metaphysical necessity. For example, water ontologically depends on hydrogen and oxygen. That is to say, the existence of hydrogen and the existence of oxygen ground the existence of water. Why then, do they not metaphysically necessitate the existence of water? The reason, I would suggest, is that hydrogen's and oxygen's existence only partially ground the existence of water. It is only together with something else, perhaps facts regarding their combination, that we have the full grounds of water. It is only with the 
existence or obtaining of the full grounds that we should expect the metaphysical necessitation of the grounded. Thus, we can explain this apparent difference in connection to metaphysical necessity between ontological dependence and grounding; many claims of ontological dependence only mention the partial grounds of the ontological dependee. Once the full grounds are given, the existence of the ontological dependee is metaphysically necessitated.

So, in the case of my existence ontologically depending on my parents' existence; my parents' existence is the partial ground for my existence, but a host of other things had to be the case- - their meeting, for one-for the fact of my existence to have been metaphysically necessitated. This solution also has the added benefit of assuaging Tahko and Lowe's worry regarding the connection between explanation and grounding, and explanation and ontological dependence. To reiterate, their worry was that citing the existence of hydrogen and oxygen, though both the ontological dependees of water, does not constitute a metaphysical explanation of the sort commonly associated with grounding: '[E]ven though the existence of water depends on the existence of hydrogen and oxygen, it does not seem to be the case that the existence of hydrogen and oxygen explain the existence of water. Rather, what explains the existence of water is the ability of hydrogen and oxygen atoms to form molecules (even though this is rather simplified). So it seems that not all relations of ontological dependence can be grounding relations in the usual sense' (Tahko and Lowe 2016: $\mathbb{5} 5$ ).

There is no reason to think, however, that citing only the existence of the partial grounds of anything should furnish us with an adequate metaphysical explanation. Though the existence of hydrogen and oxygen does ground the existence of water, it does so only partially. Were the full grounds of water to be given, we would have an explanation of the existence of water. The full grounds might include, as Tahko and Lowe helpfully suggest, some fact regarding the ability of hydrogen and oxygen to combine so, for example. It is much easier to see, then, why citing the existence of ontological dependees might not constitute a good explanation: for the reason that, in most cases, we have only cited half the explanation.

One might worry here: it seems implausible to claim that water itself ontologically depends on some fact of how hydrogen and oxygen combine such to make water. Indeed, such a fact would not seem to concern existence, which I have assumed is the hallmark of ontological dependence. We should reserve 'ontological dependence' as a term for instances of dependence like that that holds between hydrogen and oxygen, and water, so says the objector. I am happy to concede that some instances of ontological dependence will only ever be instances of merely partial grounding. I do not think this is necessarily problematic. That is to say, extraneous facts such as [how hydrogen and oxygen combine to make water], though they might also partially ground the existence of water alongside facts concerning the existence of hydrogen and oxygen, need not be said to be among the ontological dependees of water. Cases of ontological dependence may sometimes simply be instances of merely partial grounding (rather than, say, there being hidden or suppressed ontological dependees in those cases). Note my claim was that instances of grounding which concern existence solely are instances of ontological dependence; an instance of grounding which concerns only the 
existence of the grounds or the grounded exclusively (that is, the existence of one, rather than both) need not be thought of as an instance of ontological dependence. Those who are more amenable to facts other than those concerning existence standing in relations of ontological dependence might find Schnieder's analysis interesting (2006).

\section{Third Problem for Identification}

Rydéhn also presents another problem case for identifying grounding with ontological dependence: assume that [Caesar is seeing maroon] is grounded by some fact of the form [Caesar's brain is at state $N$ ], but the former fact is never grounded by Caesar's brain (the biological object). [Caesar is seeing maroon] might be said to ontologically depend on Caesar's brain the object, despite its not being grounded by Caesar's brain (Rydéhn 202I: I249). This obviously poses a problem for those who would claim grounding is ontological dependence.

How might one challenge the example? I do not think a general response tackling the class of similar cases will be forthcoming, mainly because I cannot see any general feature we might isolate from the example that would be common to multiple cases. The only recourse then seems to be to tackle the example itself.

First of all, then, we can challenge it on the basis that it requires committing to the idea of facts ontologically depending. Ontological dependence, as I have shown, is usually characterized by being the relation at issue when something depends for its existence on something else. Given that facts are not the sort of things that existrather, they obtain - we might challenge the claim that it is a genuine instance of ontological dependence in the first place. Ontological dependence concerns the relation of grounding holding between existence facts; it does not concern the existence of facts themselves, as I have characterized it.

If one does think facts enter into relations of ontological dependence, one could simply bite the bullet and hold that [Caesar is seeing maroon] is grounded by Caesar's brain. As long as one is a pluralist about the relata of grounding, there are no principled reasons for excluding Caesar's brain from entering into grounding relations. Furthermore, Caesar's brain looks like a good candidate for (partially) grounding [Caesar is seeing maroon], anyway.

One might challenge the claim that Caesar's brain does ground [Caesar is seeing maroon] on the basis that Caesar's brain does not metaphysically necessitate that [Caesar is seeing maroon]. We can respond in two, now familiar, ways. Firstly, if there are worlds in which [Caesar is seeing maroon] exists and Caesar's brain does not, it does not look like [Caesar is seeing maroon] ontologically depends on Caesar's brain anyway. Necessary coexistence, if not the whole story required for an analysis of ontological dependence, as our rejection of purely modal analyses showed, is surely still a key component of ontological dependence. We might, then, argue on this basis that this is not a genuine instance of ontological dependence, and so we can ignore it. It is worth noting this response is open only to those who hold a factive conception. On the non-factive conception, this response will not work-merely possible instances of grounding will still count as veridical instances of grounding, and therefore necessary coexistence is not a 
requisite aspect of ontological dependence. Secondly, we might account for Caesar's brain not metaphysically necessitating [Caesar is seeing maroon] in terms of partial grounding: Caesar's brain only partially grounds [Caesar is seeing maroon] and we must supplement this partial ground with facts such as [Caesar's brain is at state $N$ ] to fully ground [Caesar is seeing maroon]. When the full grounds of [Caesar is seeing maroon] are present, it is indeed metaphysically necessitated.

The example might also be understood in different terms. We might, for example, understand [Caesar is seeing maroon] in terms of the existence of a particular quale (namely, that experienced by Caesar when he sees maroon). Cast in these terms, the example would seem much less problematic, as it seems like a straight existence fact being grounded by an entity.

\section{Conclusion: Ontological Dependence}

I have here only contrasted an account of ontological dependence in terms of grounding with an account of ontological dependence in modal existential terms. There are far more ways of defining ontological dependence-essentialist accounts of ontological dependence, for example (Tahko and Lowe 2016: $\mathbb{4}$ ) - and other approaches might be superior to an account in terms of grounding. However, my aim was not to show that grounding is the best notion with which to cash out the notion of ontological dependence. It was only to show that, firstly, some of the objections to doing so are not as forceful as they might first appear, and secondly, indeed, it is stronger than modal existential analyses, at any rate.

JACK CASEY

DELFT UNIVERSITY OF TECHNOLOGY j.j.casey@tudelft.nl

\section{References}

Armstrong, David Malet. (I997) A World of States of Affairs. Cambridge: Cambridge University Press.

Audi, Paul. (20I2) 'Grounding: Toward a Theory of the "In-Virtue-Of” Relation'. Journal of Philosophy, 109, 685-7I I.

Barnes, Elizabeth. (2018) 'Symmetric Dependence'. In Ricki Bliss and Graham Priest (eds.), Reality and Its Structure (Oxford: Oxford University Press), 50-69.

Bliss, Ricki, and Kelly Trogdon. (2016) 'Metaphysical Grounding'. In Edward N. Zalta (ed.), The Stanford Encyclopedia of Philosophy. https://plato.stanford.edu/archives/winzor6/entries/ grounding/.

Cameron, Ross P. (20I0) 'From Humean Truthmaker Theory to Priority Monism'. Nô̂s, 44, I $78-98$.

Clark, Michael J. (201 5) 'A Puzzle about Partial Grounding'. Thought, 4, I89-97.

Clark, Michael J., and David Liggins. (20I2) 'Recent Work on Grounding'. Analysis, 72, 8 I 2-23.

Corkum, Phil. (2016) 'Ontological Dependence and Grounding in Aristotle'. Oxford Handbooks Online in Philosophy. https://www.doi.org/I0.1093/oxfordhb/97801999353 I4.013.3 I.

Correia, Fabrice. (2008) 'Ontological Dependence'. Philosophy Compass, 3, IOI 3-32.

Correia, Fabrice. (2010) 'Grounding and Truth-Functions'. Logique et Analyse, 53, 25 I-79.

Dasgupta, Shamik. (20I4) 'On the Plurality of Grounds'. Philosophers' Imprint, I 2, article no. 20. http://hdl.handle.net/2027/spo.352I354.00I4.020. 
Fine, Kit. (20I2) 'Guide to Ground'. In Fabrice Correia and Benjamin Sebastian Schnieder (eds.), Metaphysical Grounding: Understanding the Structure of Reality (Cambridge: Cambridge University Press), 37-80.

Jenkins, Carrie S. I. (20I I) 'Is Metaphysical Dependence Irreflexive'. Monist, 94, 267-76.

Lamarque, Peter. (2010) Work and Object: Explorations in the Metaphysics of Art. Oxford: Oxford University Press.

Leuenberger, Stephan. (20I4) 'From Grounding to Supervenience?' Erkenntnis, 79, 227-40.

Leuenberger, Stephan. (2020) 'The Fundamental: Ungrounded or All-Grounding?' Philosophical Studies, I77, 2647-69.

McLaughlin, Brian, and Karen Bennett. (2018) 'Supervenience'. In Edward N. Zalta (ed.), The Stanford Encyclopedia of Philosophy. https://plato.stanford.edu/archives/spr2oI 8/entries/ supervenience/.

Rosen, Gideon. (2010) 'Metaphysical Dependence: Grounding and Reduction'. In Bob Hale and Avi Hoffman (eds.), Modality: Metaphysics, Logic, and Epistemology (Oxford: Oxford University Press), I09-36.

Rydéhn, Henrik. (202I) 'Grounding and Ontological Dependence'. Synthese, I98, S I 23 I-56.

Schaffer, Jonathan. (2009) 'On What Grounds What'. In David Chalmers, David Manley, and Ryan Wasserman (eds.), Metametaphysics: New Essays on the Foundations of Ontology (Oxford: Oxford University Press), 347-83.

Schaffer, Jonathan. (20I2) 'Grounding, Transitivity, and Contrastivity'. In Fabrice Correia and Benjamin Schnieder (eds.), Metaphysical Grounding: Understanding the Structure of Reality (Cambridge: Cambridge University Press), I22-38.

Schnieder, Benjamin Sebastian. (2006) 'A Certain Kind of Trinity: Dependence, Substance, Explanation'. Philosophical Studies, I29, 394-4I9.

Schnieder, Benjamin Sebastian. (20II) 'A Logic for "Because"'. Review of Symbolic Logic, 4, 445-65.

Simons, Peter. (1987) Parts: A Study in Ontology. Oxford: Clarendon Press.

Skiles, Alexander. (20I4) 'Against Grounding Necessitarianism'. Erkenntnis, 80, 7I7-5I.

Takho, Tuomas, and E. Jonathan Lowe. (20I5) 'Ontological Dependence'. In Edward N. Zalta (ed.), The Stanford Encyclopedia of Philosophy. https:/plato.stanford.edu/archives/spr2o I $5 /$ entries/ dependence-ontological/\#OntDepMetGro.

Tahko, Tuomas, and E. Jonathan Lowe. (2016) 'Ontological Dependence’. In Edward N. Zalta (ed.), The Stanford Encyclopedia of Philosophy. https://plato.stanford.edu/archives/winzor 6/entries/ dependence-ontological/.

Trogdon, Kelly. (2013) 'Grounding: Necessary or Contingent?' Pacific Philosophical Quarterly, 94, $465-85$.

Witmer, D. Gene, William Butchard, and Kelly Trogdon. (2005) 'Intrinsicality without Naturalness'. Philosophy and Phenomenological Research, 70, 326-50. 